contrast differences in PAA levels between the dizygous twin pairs were statistically significant. This suggests the existence of genetic differences between PAA levels.

The factor analysis isolated ro factors which accounted for $73 \mathrm{p}$. roo of the variance of the $2 \mathrm{r}$ amino acids and amines. The communalities of individual AA varied between $50 \mathrm{p}$. 100 and $94 \mathrm{p}$. 100 whereby $45 \mathrm{p}$. I 100 of the variance was accounted for by four factors. No general factor appeared but a number of group factors of which the 4 more important ones accounted for $45 \mathrm{p} .100$ of the sum of squares.

$$
\begin{aligned}
& \text { Factor } \text { I.69 } \mathrm{Hi}+.56 \mathrm{Thre}+.75 \mathrm{Se}+.78 \mathrm{Pro}+.5 \mathrm{I} \text { Meth }-.76 \mathrm{HST}+.35 \text { Cit } \\
& 2.40 \mathrm{Va}+.78 \mathrm{Ile}+.87 \mathrm{Lau}+.39 \mathrm{Tyr}-.35 \mathrm{Cit} \\
& 3.68 \mathrm{Glu}+.66 \mathrm{Cy}+.44 \mathrm{Va} \\
& 9.57 \mathrm{Thre}+.7 \mathrm{Ty}+.7 \mathrm{I} \mathrm{Pal}+.57 \mathrm{Tau}
\end{aligned}
$$

Factor load of less than .3I are not given but included in computation of the factor values of individual animals.

FA I has positive loads of essential and growth promoting AA. It appears to reflect the status of the AA-pool.

FA 2 is related to branched chain AA, FA 9 concerns Tyrosin and Phenylalanin, and also Taurin and Threonine.

All factors show highly significant differences between young steers and calves. Differences between milk - fattened and early weaned calves appear significant only for FA 2 and FA 9.

Values from calves were submitted to an analysis of variance (pairs, animals/pairs, residual). All meansquares for pairs and animals/pairs (with the exception for FA 9) are larger than the succeeding ones, some of those for pairs are highly significant. Genetic differences appear likely and are more pronounced than for individual AA.

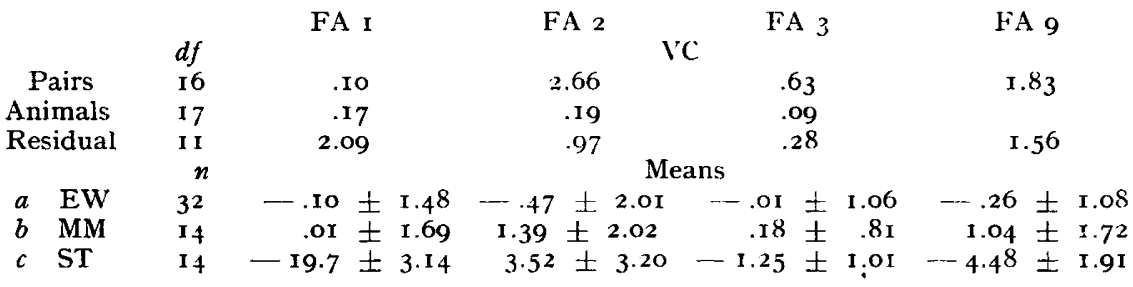

\title{
THE INFLUENCE OF NUTRITION \\ (ENERGY AND DIGESTIBLE CRUDE PROTEIN) \\ ON SOME BI,OOD PARAMETERS, \\ HEALTH AND FERTILITY IN LATE PREGNANT MILKING COWS
}

K. H. Lotthammer, F. E. Farries. - Klinik für Geburtshilfe und Gynäkologie des Rindes (im Richard-Götze-Haus) der Tievärztlichen Hochschule, Hannover, BRD.

In einem definierten Versuch wurde der Einfluss einer unterschiedlichen Versorgung an Energie und verdaulichem Rohprotein in der Hochträchtigkeit auf verschiedene Metaboliten im Blutplasma und auf die Gesundheit und Fruchtbarkeit von Milchkühen untersucht. Jeweils 3o Schwarzbunte Milchkühe wurden in den letzten Io Wochen der Trächtigkeit für die Erhaltung und $18 \mathrm{~kg}$ FCM (Versuchsgruppe I - überversorgt) bzw. für die Erhaltung und 2 kg FCM (Versuchsgruppe II - unterversorgt) hinsichtlich Energie und verdauliches Rohprotein versorgt.

In den letzten 5 Wochen der Trächtigkeit und bis in die 4 . Woche p.p. wurden wöchentlich die Gehalte an anorganischem Phosphor, Gesamt-Protein, Gesamt-Billrubin, Gesamt-Cholesterin sowie die Aktivität der Glutamat-Oxalacetat-Transaminase (GOT) im Blutplasma bestimmt. Nach dem Abkalben wurden die Kühe regelmass klinisch-gynäkologisch rektal und vaginal wöchentlich untersucht, bis zur festgestellten Trächtigkeit.

Bereits 2 Wochen a.p. konnte in der nochversorgten Gruppe 1 eine Stoffwechselbelastung festgestellt werden. (erhöhte GOT-Werte). Nach dem Abkalben bestand in der Versuchsgruppe I eine starke Stoffwechselbelastung, die sich in crhöhten GOT- und Bilirubin-Werten sowie in stark erniedrigten P-Gehalten ausdrückte.

In der hochversorgten Gruppe I war der Verlauf der Puerperiums stark gestört (verzögerte Uterusinvolution, eitrige Endometritiden), es traten mehr Stoffwechselstörungen (Gcbärparese, 
Azctonurie) und in späteren Verlauf des Puerperiums mehr Fruchtbarkeitsstörungen (eitrige Genitalkatarrhe, Ovarialzysten, niedrige Erstbesamungsergebnisse) auf. Entsprechend waren auch die Abgänge wegen Sterilität und Stoffwechselstörungen in der Versuchgruppe 1 höher als in der Gruppe II. Der Verlauf der Erkrankungen war in der Gruppe I schlechter und erforderte mehr tierärztliche Behandlungen.

Die Versuchsergebnisse zeigen dass eine Uberversorgung an Energie und verdaul. Ronprotein in der Hochträchtigkeit zu einer starken Beeinträchtigung der Gesundheit und Fruchtbarkeit p.p. zur Folge haben kann. Der negative Einfluss kann sich bis etwa + Wochen p.p. auswirken. Die Versorgung sollte deshalb in der Hochträchtigkeit für die Erhaltung und 5 bis maximal to $\mathrm{kg}$ Milch bemessen sein, um einen negativen Einfluss auf die Gesundheit und Fruchtbarkeit zu vermeiden.

\section{UNTERSUCHUNGEN ZUR ACETONÄMIE BEI DER MILCHKUH}

E. FARRIES, BRD.

Acetonämie ist eine Störung im Energiestoffwechsel bei Hochleistungskühen zu Laktationsbeginn. Sie steht im Zusammenhang mit einer unvollständigen Metabolisierung von Fettreserven, die während der Gravidität angelegt wurden. Besondere Belastungen im Glukose-Stoffıvechsel werden dabei beobachtet.

Versuchsanstellung : Durch gezielte Fütterungsmassnahmen wurden bei 6 o Hochleistungskühen in der Spätgravidität (letzte ro Wochen a.p.) unterschiedliche Energiereserven angelegt, die dann zu Laktationsbeginn (erste ro Wochen p.p.) einer differenzierten Nutzung unterlagen.

Versuchsergebnisse : Gewichtsentwicklung : Die Gewichtsentwicklung als Mass für den V'erlauf von Reservebildung und Mobilisierung steht in deutlicher Abhängigkeit von der Nährstoffzufuhr. Ein Einfluss auf die Kälbergewichte ist kaum kaum zu erkennen.

Glukose : In der Gravidität bestehen keine fütterungsabhängigen I'nterschiede in der Blut-Glukose-Konzentration. $\mathrm{Zu}$ Laktationsbeginn liegt der Glukose-Spiegel bei den vorher hochversorgten Tieren deutlich niedriger ( $\mathrm{Ca} 40 \mathrm{mg} / \mathrm{roO} \mathrm{ml}$ ) als bei den niedrigversorgen (Ca $50 \mathrm{mg} / \mathrm{I}$ oo $\mathrm{ml}$ ). Ketonkörper: Die Konzentration an Ketonkörpern (Aceton, Acetacetat, $\beta$-Hydroxybutyrat) ist der den Glukose entgegengesetzt. Tiere mit hoher Energiereserve und verstärkter Mobilisierung erreichen hohe Werte (30 $\mathrm{mg} / \mathrm{roO} \mathrm{ml}$ ) gegenüber denen mit geringem Depot ( $14 \mathrm{mg} / \mathrm{roO} \mathrm{ml}$ ).

Erhöhte Reservebikdung zum Zeitpunkt der Spätgravidität führt zu eine verstärkten Belastung des Energiestoffwechsels am Laktationsbeginn.

\section{VERWENDUNG VON STOFFWECHSELPARAMETERN} ZUR BEURTEILUNG VON HALTUNGSSYSTEMEN BEIM RIND

B. Oldigs, D. Smidt, H. J. Langholz, U. Meyer-Otting und G. Flitz-Pries. - B B BD.

Es wird über Untersuchungen an drei Kuhpopulationen in mehreren Haltungssystemen (Anbindestall, Kombibuctenstall, Tieflaufstall, Weide) berichtet. An Hand der ersten Versuchsergebnisse, die u.a. Stoffwechselparameter zum Inhalt hatten, wird diskutiert, ob bzw. welche Parameter zur Bewertung der Haltungssysteme geeignet sein können.

\section{THE INTERRELATIONSHIP BETWEEN METABOLIC PARAMETERS IN BLOOD SERUM OF CATTI,E AND FERTILITY}

\section{H. Sommer. - Universität Bonn, Institute of Anatomy and Physiology and Hygiene, BDR.}

Zwischen dem Gesamtcholesterin und der GOT im Blutserum bestehen Beziehungen zu verschiedenen Fruchtbarkeitsstörungen und damit zur Fruchtbarkeitsleistung. Die Verwendung der oben genannten und ähnlicher Parameter als Hilfsmerkmale zur Selektion auf Fruchtbarkeit 